\title{
Analysis of the Collapse of Long-Span Reticulated Shell Structures Under Multi-Dimensional Seismic Excitations
}

\author{
Ming-Fei Yang \\ Key Laboratory of C\&PC Structures of the Ministry of Education, Southeast University, Nanjing 210096, China \\ School of Civil Engineering, Anhui University of Science and Technology, Huainan 232001, China \\ Zhao-Dong Xu, Xing-Huai Huang and Han-Hu Ye \\ Key Laboratory of C\&PC Structures of the Ministry of Education, Southeast University, Nanjing 210096, China
}

\begin{abstract}
(Received 1 August 2012; accepted 25 April 2013)
The collapse processes of three typical long-span reticulated shell structures were simulated using nonlinear dynamic finite element analysis under strong seismic excitations. The plastic kinematic hardening model, which considers failure stain, was adopted for simulating steel. Both geometric and contact nonlinearities were considered in this study. The three failure states-i.e., dynamic local bucking, dynamic overall buckling, and whole collapse-were identified in accordance with the analysis results. Taking the Schwedler reticulated shell structure as an example, seismic waves were applied to the structure in three directions. The critical loads were obtained by the incremental dynamic analysis method (IDAM), and some critical state indices were obtained according to the dynamic responses. The results showed that all the critical indices need to be considered simultaneously in order to judge the dynamic collapse states.
\end{abstract}

\section{NOMENCLATURE}

$\begin{array}{lll}\mathbf{M}, \mathbf{C}, \mathbf{K} & - & \begin{array}{l}\text { Mass matrix, damping matrix and } \\ \text { stiffness matrix of long-span retic- } \\ \end{array} \\ & \text { ulated shell structures } \\ \ddot{\mathbf{u}}(t), \dot{\mathbf{u}}(t), \mathbf{u}(t) & - & \begin{array}{l}\text { Acceleration, velocity, and dis- } \\ \text { placement array }\end{array} \\ \mathbf{P}(t) & - & \text { Excitation vector } \\ \Delta t, \Delta t_{\mathrm{cr}} & - & \text { Time step and critical time step } \\ \omega_{\max } & - & \text { Largest circular frequency } \\ T_{\min } & - & \text { Smallest fundamental period } \\ T & - & \text { Period of structures }\end{array}$

\section{INTRODUCTION}

Long-span reticulated shell structures currently provide a widely-used structural solution to the problem of spanning large uninterrupted distances. Due to the increase in the size of such structures, it is imperative to make intensive studies about the plastic properties and the development law of plastic displacement, the buckling behaviours of long-span shell structures, and the collapse mechanism caused by strong earthquakes. ${ }^{1}$ Nevertheless, it is difficult to investigate the collapse mechanism of long-span reticulated shell structures when the member is in the plastic state. However, finite element technology can solve the problem by considering the nonlinear material and geometric factors, and this technology has been used widely in analysis.

In recent years, a great deal of research has been focused on assessing the seismic response of long-span spatial structures. $^{2-6}$ The strength of reticulated spherical domes has been generally associated with the inelastic buckling of slender members, and more and more partially restrained connections between structural members. This conclusion was verified by Battista et al. in 2001. ${ }^{7}$ The elastic-plastic dynamic response of single-layer reticulated shells under strong earthquake excitation, static, and dynamic loads was analysed by $\mathrm{Li}$ and Chen in 2003-2004. ${ }^{8}$ Through the numerical example, the stability was influenced by the geometric parameters, which define the mesh of elements. In 2003, the seismic time history responses of long-span reticulated shell structures were obtained by Guo and Shen using ANSYS software, and the failure mechanism subjected to severe earthquakes was first described. ${ }^{9}$ Taniguchi ${ }^{10}$ quantified the relationships between the dynamic characteristics of lattice structures and those of earthquakes. In the next year, the shaking table tests of vertical and horizontal directions on long-span reticulated shell structures, with viscoelastic multi-dimensional earthquake isolation and mitigation devices, were studied by $\mathrm{Xu}^{1{ }^{11-13}}$ And after that, a computationally effective method for evaluating the dynamic buckling and post buckling of thin composite shells was verified by Chamis in 2010. ${ }^{14}$ However, as of yet there is still not a unified standard to describe the dynamic collapse process and determine the collapse index of long-span reticulated shell structures.

In order to judge the dynamic collapse states and obtain the dynamic collapse indices of long-span reticulated shell structures, the collapse process was numerically analysed using ANSYS/LS-DYNA software. Based on the numerical analysis results, the dynamic collapse states were described in detail. Finally, the collapse indices were proposed by the incremental dynamic analysis method (IDAM). 